\title{
Análise dos aspectos éticos da pesquisa em seres humanos contidos nas I nstruções aos Autores de 139 revistas científicas brasileiras
}

\author{
T. Sardenberg, S. S. Müller, H. R. Pereira, R. A. de Oliveira, W. S. Hossne
}

Departamento de Cirurgia e Ortopedia da Faculdade de Medicina de Botucatu, U niversidade Estadual Paulista - U nesp, B otucatu, SP.

RESUMO - ОвJ ETIVo. Estudar as normas relativas à ética da pesquisa em seres humanos contidas nas Instruções aos Autores de revistas científicas brasileiras.

Material e Métodos. As I nstruções aos Autores de 139 revistas científicas brasileiras das áreas de medicina, biomedicina, enfermagem, odontologia e ciências gerais foram analisadas com relação às suas recomendações sobre os aspectos éticos.

Resultados. Das 139 revistas estudadas, 110 (79,1\%) não fazem referências aos aspectos éti cos; $17(12,2 \%)$ exigem aprovação prévia pela Comissão de É tica; três (2,1\%) fazem referência à Declaração de Helsinque; uma $(0,7 \%)$ recomenda adotar o consentimento esclarecido; cinco (3,5\%) seguem orientações dos requisitos uniformes para manuscritos submetidos a revistas biomédicas e três $(2,1 \%)$ seguem princípios, normas e padrões

\section{NTRODUÇÃO}

O Tribunal de Nuremberg, que julgou os crimes de guerra da Segunda Guerra Mundial em 1947, elaborou o Código de Nuremberg ${ }^{1}$, estabel ecendo dez tópicos que os médicos devem seguir quando realizam experimentos em seres humanos. O primeiro, maior e mais detal hado, explicita que "O consentimento voluntário dos sujeitos humanos é absolutamente necessário". Não há referências que protocolos de pesquisa em seres humanos devem ser aprovados previamente por comissão independente e nem referências relativas à publicação dos resultados desses estudos.

A Declaração de Helsinque da Associação Médi ca Mundial, de 1964, (revisada diversas vezes, sendo a última edição aprovada na 48a Assembléia Geral na República da África do Sul em 1996)², afirma que protocol os de pesquisa em seres humanos devem ser analisados por comitê independente do investigador e que "relatos de experimentação fora dos princípios desta Declaração não devem ser aceitos para publicação". éticos não especificados. Nas 29 revistas que fazem referências à ética, as exi gências são solicitadas sob as seguintes formas: $15(51,7 \%)$ exigem a inclusão da informação no texto do artigo; duas $(6,8 \%)$ pedem carta assinada pelos autores; uma $(3,4 \%)$ solicita cópia da autorização da Comissão de Ética; uma $(3,4 \%)$ afirma supor que o autor cumpriu as exigências e dez $(34,4 \%)$ não fazem qualquer referência específica.

Conclusões. Os resultados do estudo indicam que na maioria das revistas científicas brasileiras há pouca preocupação em relação aos aspectos éticos da pesquisa em seres humanos, contidas nas Instruções aos Autores. Nas revistas que fazem referência aos aspectos éticos, as exigências são muito variáveis.

Unitermos: Ética. Bioética. Pesquisa. Editoração. Periódicos.

O Código de Ética Médica Brasileiro³, de 1988, contém capítulos referentes à pesquisa médica e ao trabal ho científico. No que diz respeito à pesquisa médica (nove artigos), faz referências ao consentimento e à necessidade de que os protocolos de pesquisa sejam submetidos à aprovação e acompanhamento por comissão independente do pesqui sador. No capítul o sobre trabal ho ci entífico, não há referência aos aspectos éticos para fins de publicação de resultados de estudos em seres humanos. O Código de Ética dos profissionais de enfermagem ${ }^{4}$ e o Código de Ética Odontológica ${ }^{5}$ assinalam a necessidade do consentimento informado para a realização de pesquisas em seres humanos.

O Consel ho Nacional de Saúde do Ministério da Saúde (CNS-MS) editou, em 1988, a Resolução $n^{\circ}$ $1 / 88^{6}$ que, entre diversos itens, estabelece a necessidade do "consentimento pós-informação" e exige que os protocolos de pesquisa sejam aprovados por Comi têdeÉ tica independente do pesquisador, sem referência aos aspectos éti cos relacionados à publicação dos resultados das pesquisas em 


\begin{tabular}{|lr|}
\hline $\begin{array}{l}\text { Tabela 1 - Freqüências absoluta e relativa de referências éticas } \\
\text { da pesquisa em seres humanos contidas nas Instruções aos } \\
\text { Autores de revistas científicas brasileiras. }\end{array}$ \\
\hline Comitê ou Comissão de Ética & $17(12,2 \%)$ \\
Declaração de Helsinque ou Código de Nuremberg & $3(2,1 \%)$ \\
Consentimento Esclarecido & $1(0,7 \%)$ \\
Requisitos Uniformes & $5(3,5 \%)$ \\
Princípios / Normas / Padrões Éticos & $3(2,1 \%)$ \\
Sem Referências Éticas & $110(79,1 \%)$ \\
& $n=139(100 \%)$ \\
\hline
\end{tabular}

Tabela 2 - Freqüência de referências éticas da pesquisa em seres humanos contidas nas Instrução aos Autores de revistas científicas brasileiras, classificadas de acordo com as especialidades.

\begin{tabular}{|lcc|}
\hline & Com Ref. Éticas & Sem Ref. Éticas \\
Cirurgia (16) & 8 & 8 \\
Clínica Médica (14) & 5 & 9 \\
Pediatria (4) & 2 & 2 \\
Psiquiatria (4) & 0 & 4 \\
Medicina Geral (26) & 7 & 19 \\
Medicina Social/Ocupacional (7) & 1 & 6 \\
Oncologia (3) & 0 & 3 \\
Especialidades (5) & 1 & 4 \\
Enfermagem (5) & 2 & 3 \\
Miscelânia (11) & 0 & 11 \\
Odontologia (44) & 3 & 41 \\
\hline Frequência absoluta & 29 & $(79,1 \%)$ \\
Frequência relativa & & \\
$\mathrm{n}=139$ & $(20,8 \%)$ & \\
\hline
\end{tabular}

seres humanos. Em 1996, o CNS-MS aprovou a Resol ução 196/967, que incorpora vários conceitos da bi oética e mantém o consentimento do indivíduo e a necessidade de aprovação prévia por Comi tê deÉ tica. A Resol ução 196/96 não esti pula regras nesse sentido, contudo, estabelece que os resultados da pesquisa em seres humanos devem ser tornados públicos, sejam el es favoráveis ou não.

Apesar de todos esses documentos serem referências oficiais para os pesquisadores, a preocupação com os aspectos éticos da pesquisa em seres humanos no Brasil, principalmente em relação à aprovação por comissões ou comitês de ética, sofreu grande impacto com a exigência de diversas revistas científicas internacionais, notadamente as de língua ingl esa, por somente aceitarem, para análise e possível publicação, estudos cujos protocolos tenham sido aprovados previamente por comissões institucionais.

A publicação ou não de artigos considerados eti camente inadequados é polêmica antiga no meio científico e, no entanto, permanece extremamente atual ${ }^{8,13}$. Houve, contudo, nítida mudança no enfoque, uma vez que não mais se debate a publicação ou não de arti gos clara e generi camente consi derados não-éticos, mas sim se as revistas científicas devem publicar pesquisas que não incluem, na sua execução, o consentimento informado ou não foram analisadas e aprovadas por comitês de ética institucionais.

Nas últimas duas décadas tem havido, entre os editores de revistas científicas internacionais, constante preocupação em estabel ecer orientações padronizadas para a elaboração de manuscritos a serem submetidos para análise, objetivando publicação, sendo fruto desse esforço o Comitê I nternacional de Editores de Revistas Médicas, o qual edita os "Requisitos uniformes para manuscritos submetidos às revistas biomédicas" (RUMSRB), atual mente seguido por mais de 500 revistas, inclusive no Brasil 14-16. As orientações dos RUMSRB fazem referência explícita à Declaração de H el sinque e aos comitês responsáveis por experimentação humana (regional ou institucional). É solicitado, ainda, às revistas que concordam com os RUMSRB, que o documento seja citado em suas Instruções aos Autores.

Atualmente, grande parte das revistas científicas da área de medicina e biomedicina de língua inglesa fazem referência aos aspectos éticos da pesquisa em seres humanos em suas Instruções aos Autores ${ }^{17}$. Não foi possível encontrar na literatura científica brasileira publicações que estudassem o impacto e a presença dos paradigmas atuais da bioética na prática editorial. Diante desse quadro, decidimos analisar, a partir das Instruções aos Autores de revistas científicas brasileiras das áreas de medicina, biomedicina, enfermagem, odontologia e ciências gerais, os aspectos éticos relacionados às pesquisas em seres humanos.

\section{MATERIAL E MÉTODOS}

As seç̧ões de Instruções aos Autores de 139 revistas científicas brasileiras de medicina, biomedicina, enfermagem, odontol ogia e ci ências gerais foram analisadas em relação às orientações éticas das pesquisas em seres humanos de modo semel hante ao estudo de Amdur e Biddl $\mathrm{e}^{17}$.

As revistas foram agrupadas, sempre que possível, de acordo com as especialidades médicas. As revistas de doenças infecciosas, por exemplo, foram classificadas em Clínica Médica e as de Ginecologia e Obstetrícia como da área de Cirurgia. Revistas sem área específica foram catalogadas 


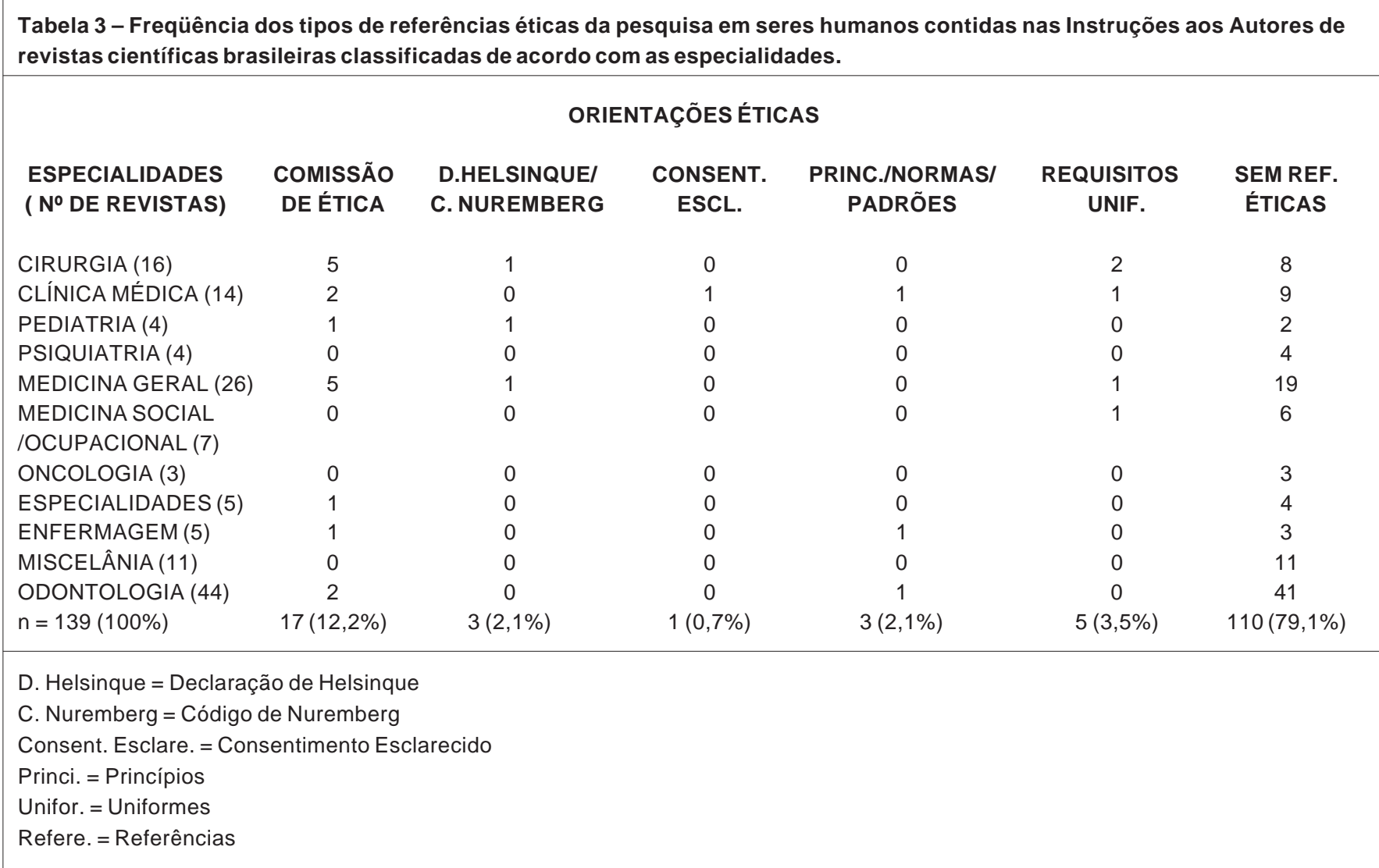

como Medicina Geral e as publicações de biomedicina, bi ol ogia e ciências gerais foram agrupadas em Miscelânia. Todas as revistas de Odontologia foram incluídas em um mesmo grupo.

Em relação aos aspectos éticos das orientações contidas nas Instruções aos Autores, principalmente na parte relacionada à pesquisa em seres humanos, as revistas foram classificadas em seis categorias:

Comissão ou Comitê de Ética - revistas que fazem referência à necessidade de aprovação e/ou análise dos trabalhos por Comissão ou Comitê de Ética da instituição onde o estudo foi realizado, independentemente de haver outras recomendações. Por exemplo, a Revista do Col égi o Brasileiro de Cirurgi ões, classificada nesse grupo, cita: "Toda matéria relacionada à investi gação humana e à pesquisa animal deve ter aprovação prévia da Comissão de Ética da Instituição onde o trabalho foi realizado, de acordo com as recomendações da Resol ução de Helsinque e as Normas I nternacionais de Proteção aos Animais".

Declaração de Helsinque - revistas em que não há referência à Comissão ou Comitê de Ética da instituição onde o estudo foi realizado, porém, citam a Declaração de Helsinque, independentemente de haver referências a outros aspectos éti- cos. Classificada nesse grupo, a Revista do Hospital de Clínicas de Porto Alegre cita, nas suas Instruções aos Autores, além da Declaração de Hel sinque, a Resolução 196/96 do Consel ho Naci onal de Saúde e as resoluções normativas sobre pesquisa do Hospital de Clínicas de Porto Alegre.

Consentimento do paciente - revistas em que a única referência aos aspectos éticos da pesquisa em seres humanos é a solicitação de obtenção do consentimento do paciente.

Princípios/ Normas/ Padrões Éticos - revistas que fazem referência genérica ao respeito à ética, princípios, normas, padrões etc. Por exemplo, a Revista Brasileira de Reumatologia afirma: "Os padrões científicos e éticos são básicos para a aceitação de qual quer tipo de trabalho".

Requisitos Uniformes -revistas em que há referência direta ou indireta às orientações dos "Requisitos uniformes para manuscritos submetidos à revistas biomédicas", proposto pelo "Comitê I nternacional de Editores de Revistas M édicas". Não foram classificadas nesse grupo as revistas que fazem referência aos RUMSRB somente em relação à apresentação das referênci as bi bli ográficas e outros aspectos técnicos.

Sem Orientação Ética - revistas que não fazem qualquer referência aos aspectos éticos relaciona- 


\begin{tabular}{|lr|}
\hline $\begin{array}{l}\text { Tabela 4 - Freqüência das maneiras de como as Instruções } \\
\text { aos Autores de revistas científicas solicitam que as informa- } \\
\text { ções referentes aos aspectos éticos das pesquisa em seres } \\
\text { humanos sejam fornecidas. }\end{array}$ \\
\hline Incluir no texto do artigo & $15(51,7 \%)$ \\
Carta assinada pelos autores & $2(6,8 \%)$ \\
Cópia da autorização da Comissão de Ética & $1(3,4 \%)$ \\
Subentendido (o autor assume a responsabilidade) & $1(3,4 \%)$ \\
Sem referências & $10(34,4 \%)$ \\
& $n=29(100 \%)$ \\
\hline
\end{tabular}

Quadro 1 - Revistas científicas que fazem referências à Declaração de Helsinque, isoladamente ou em conjunto com outras orientações, nas Instruções aos Autores.

Revista Colégio Brasileiro de Cirurgiões

Revista Brasileira de Oftalmologia

Revista do Hospital de Clínicas de Porto Alegre

Revista Brasileira de Medicina

Revista Brasileira de Medicina - Ginecologia e Obstetrícia

Revista Paulista de Pediatria

Quadro 2 - Revistas científicas que fazem referências ao consentimento do paciente, isoladamente ou em conjunto com outras orientações, nas Instruções aos Autores.

Arquivo Brasileiro de Cardiologia

Jornal Brasileiro de Nefrologia

Revista Brasileira de Medicina

Revista Brasileira de Medicina - Ginecologia e Obstetrícia

Revista Brasileira de Anestesiologia

Revista Latino Americana de Enfermagem

Revista da Pós-Graduação

Revista de Odontologia da USP

dos à pesquisa em seres humanos. Revistas que fazem referências somente aos aspectos rel acionados à privacidade dos pacientes ou que citam o termo "paciente ou casuística" no lugar de "material" também foram classificadas nesse grupo.

As maneiras como os aspectos éticos dos artigos enviados para as revistas científicas devem ser informados aos editores foram classificadas em cinco grupos:

Incluído no texto do artigo - revistas que claramente indicam que as informações sobre os aspectos éticos da pesquisa em seres humanos devem ser citadas no texto do artigo.

Carta assinada - revistas que exigem carta ou documento assinado pelo autor informando os aspectos éticos do estudo.

Cópia de autorização do Comitê ou Comissão de Ética - revistas que explicitam a exigência do envi o de cópia da autorização da Comissão de Ética da instituição onde o estudo for realizado, junto com o manuscrito da pesquisa.

Subentendido - revistas que informam nas I ns- truções aos Autores que o editor subentende que o estudo foi realizado dentro das normas, padrões ou princípios éticos, sem a exigência de qualquer outro tipo de informação.

Sem referências - revistas que não contêm nenhuma informação de como os autores dos manuscritos enviados para publicação devem informar sobre os aspectos éticos do estudo.

\section{RESULTADOS}

O resultado da análise das orientações éticas solicitadas nas Instruções aos Autores de 139 revistas científicas brasileiras da área de medicina, biomedicina, enfermagem, odontologia e ciências gerais estão sumarizados nas tabelas 1, 2, 3 e 4 e quadros 1,2 e 3 .

O número de revistas consideradas essencialmente médicas (classificadas em Cirurgia, Clínica Médica, Pediatria, Medicina Geral, Medicina Social e Ocupacional, Oncol ogia eE specialidades) éde 79 , sendo que $24(30,3 \%)$ apresentam al guma referência à ética de pesquisa em seres humanos; na área de odontologia, de um total de 44 revistas, somente três $(6,8 \%)$ apresentam orientações éticas.

Devido à grande variabilidade nas referências aos aspectos éticos da pesquisa em seres humanos nas I nstruções aos Autores, observada nas revistas estudadas, privilegiou-se, na classificação adotada, as referências relacionadas às Comissões ou Comitês de Ética.

Dentro desse contexto, várias revistas que fazem referências à Declaração de Helsinque, Código de Nuremberg e "Consenti mento esclareci do do paciente" foram classificadas no grupo "Comitêl Comissão de Ética". No entanto, devido à importância desses aspectos éticos, todas as suas citações foram avaliadas, observando-se que a Declaração de Hel sinque é citada seis vezes, o Código de Nuremberg uma e o "consentimento do paciente" oito vezes.

\section{DISCUSSÃO}

O presente estudo limitou-se à análise das I nstruções aos Autores, não sendo realizadas entrevistas com os editores e nem análises de artigos publicados nessas revistas. Trata-se, portanto, de análise parcial da política editorial em relação à éti ca desses periódi cos. No entanto, as orientações contidas na secção de I nstruções aos Autores das revistas é a primeira e, muitas vezes, a única via de comunicação entre pesquisador e editor e, portanto, a política adotada pel os periódicos, refl eti da nas Instruções aos Autores, e reveste-se de enor- 
Quadro 3 - 139 revistas científicas brasileiras classificadas de acordo com as referências aos aspectos éticos da pesquisa em seres humanos nas Instruções aos Autores.

\section{Comissão ou Comitê de Ética}

Jornal de Pneumologia

Revista de Medicina da PUCRS

Revista Colégio Brasileiro de Cirurgiões

Revista Brasileira de Ginecologia e Obstetrícia

Revista Brasileira de Otorrinolaringologia

Revista Paulista de Medicina

Jornal Brasileiro de Nefrologia

Revista Brasileira de Medicina

Revista Brasileira de Medicina - Ginecologia e Obstetrícia

Revista Brasileira de Anestesiologia

Jornal de Pediatria

Braziliam Journal of Medical and Biological Research

Revista Saúde (UF Sta Maria)

Revista Latino Americana de Enfermagem

Acta Cirúrgica Brasileira

Revista da Pós-Graduação

Revista de Odontologia

Declaração de Helsinque/Código de Nuremberg)

Revista Brasileira de Oftalmologia

Revista Paulista de Pediatria

Revista do Hospital de Clínicas de Porto Alegre

Consentimento Esclarecido

Arquivos Brasileiro de Cardiologia

Princípios / Normas / Padrões Éticos

Revista Brasileira de Reumatologia

Revista Brasileira de Enfermagem

RFO - UPF

\section{Requisitos Uniformes}

Jornal Brasileiro de Urologia

Revista de Saúde Pública

Revista Médica de Minas Gerais

Revista Brasileira de Crescimento e Desenvolvimento Humano

Revista Brasileira de Cirurgia Cardiovascular

\section{Sem Referências Éticas}

Revista da Sociedade Brasileira de Cirurgia Plástica

Revista Brasileira de Ortopedia

Acta Ortopedica Brasileira

Jornal Brasileiro de Ginecologia

Revista Brasileira de Colo-Proctologia

Revista Brasileira de Cirurgia

Revista Brasileira de Mastologia

Revista de Ginecologia \& Obstetrícia

Revista Brasileira e Latino Americana de Marca Passo e Arritmia

The Braziliam Journal of Infectious Diseases

Revista da Sociedade de Cardiologia do Estado de São Paulo

Revista Brasileira de Clínica \& Terapêutica

Revista Brasileira de Medicina Psicossomática

Revista da Sociedade Brasileira de Medicina Tropical

Revista do Instituto de Medicina Tropical de São Paulo

Revista HiperAtivo

Revista de Homeopatia

Pediatria (São Paulo)

Arquivos Brasileiros de Pediatria

Revista da Escola de Enfermagem da USP

Revista Paulista de Enfermagem

Revista Gaucha de Enfermagem

Revista de Psiquiatria Clínica

Psiquiatria Biológica

Revista ABP - APAL

Jornal Brasileira de Psiquiatria

Revista da Associação Médica Brasileira

Ars Cvrandi

A Folha Médica

Revista de Medicina

Arquivos Médicos dos Hospitais e da Faculdade de Ciências

Médicas da Sta Casa de São Paulo

Revista de Medicina do Hospital Universitário

Revista Médica do IAMSPE

latros

Salusvite
Revista de Saúde do Distrito Federal

Revista do Hospital das Clínicas FMUSP

Arquivos Brasileiros de Medicina

Anais Paulista de Medicina e Cirurgia

Arquivos Catarinense de Medicina

Revista de Ciências PUCCAMP

Medicina

Anais da Academia Nacional de Medicina

Jornal Brasileiro de Medicina

Revista Goiana de Medicina

Revista da Faculdade de Ciências Médicas da Unicamp

Mundo da Saúde

Revista do Instituto Adolfo Lutz

Cadernos de Saúde Pública

Saúde em Debate

Revista Brasileira de Saúde Ocupacional

História - Ciência - Saúde - Manguinhos

Revista Brasileira de Cancerologia

Acta Oncológica Brasileira

Oncologia Atual

Jornal Brasileiro de Patologia

Revista de Ciências Biomédicas

Revista de Ciências Farmacêuticas

Revista de Farmácia e Bioquímica da USP

Revista de Nutrição da PUCAMP

Revista Brasileira de Análises Clínicas

Braziliam Journal of Genetics

Anais da Acadêmia Brasileira de Ciências

Revista Brasileira de Biologia

Journal of the Braziliam Society of Microbiology

Revista Brasileira de Educação Médica

Anais Brasileiros de Dermatolgia

Arquivos de Neuro-Psiquiatria

Revista Brasileira de Neurologia

Arquivos de Gastroenterolgia

Revista Paulista de Odontologia

Odontologia Gaucha

Odontologia USF

Revista de Odontopediatria - Atualização e Clínica

Revista de Odontologia da UNICID

Revista de Odontologia da UNESP

Revista da Faculdade de Odontologia da UF de Pelotas

Revista da Faculdade de Odontologia da UF da Bahia

Revista de Periodontia da Sobrape

Revista Brasileira de Odontologia

Revista Científica CENBIOS

Revista do Instituto Brasileiro de Implantologia

Revista do CROMG

Revista Brasileira da Ciência Estomalógicas

Revista de Odontologia da Faculdade de Odontologia de Bauru

Revista da Faculdade de Odontologia de Lins

Revista da Faculdade de Odontologia da UFRG

Arquivos do Centro de Estudos do Curso de Odontologia

Stomatos

Revista de Odontologia do Brasil Central

Revista SPRO

Revista Goiânia de Ortodontia

Revista do Centro de Estudos da FO UERJ

Revista Dental Press de Ortodontia e Ortopedia Maxilar

Revista Odontologia de Santo Amaro

Braziliam Endodontia Journal

Dens

Jornal Brasileiro de Odontopediatria e Odontologia do Bebê Implantares

FOPLAC em Revista

Odontologia - Ensino e Pesquisa

Odontologia Clínica

Odonto Pope

Odonto

Odonto 2000

Braziliam Dental Journal

Revista APCD

Revista ABO - Nacional

Revista Ortodontia

Revista do Instituto da Saúde 
me importância na prática de publicação de artigos científicos.

Goldenberg et al. ${ }^{18}$, analisando diversos aspectos das Instruções aos Autores de 19 periódicos médicos brasileiros, salientaram a importância dessa seção na avaliação da política editorial das revistas. Não realizaram, porém, qualquer análise relativa às orientações éticas dessas revistas.

Em nossa amostra, o número de revistas que fazem referência aos aspectos éti cos da pesquisa em seres humanos nas I nstruções aos Autores foi baixo $(20,8 \%)$, mesmo com os critérios extremamente flexíveis de avaliação utilizados neste estudo.

As revistas de medi cina e enfermagem apresentaram maior preocupação com as orientações éticas do que as revistas não-médicas, classificadas nos grupos de miscelânia e odontologia, sugerindo que o debate ético da experimentação em seres humanos está mais difundido nas áreas médica e de enfermagem do que em outras áreas de ciências da saúde. $\mathrm{Na}$ área de odontologia, observa-se que as orientações éticas contidas no Código de Ética Odontológica, extremamente claras e precisas, encontram poucos reflexos na política editorial dos periódicos.

O Código de Nuremberg e a Declaração de Helsinque são documentos que fazem referência à experimentação médica em seres humanos. A resolução do CNS-MS de 1988 faz referência à pesquisa na área de saúde. Por outro lado, a atual legislação brasileira, resolução 196/96 do CNSMS, refere-se a qualquer pesquisa envolvendo seres humanos, inclusive de áreas como sociologia, economia e meio-ambiente.

Brackbill e Hellegers ${ }^{19}$ realizaram entrevistas com editores de revistas médi cas e observaram que $27 \%$ procediam à revisão ética dos artigos enviados para publicação e $21,3 \%$ exigiam que os estudos fossem previamente aprovados por Comitês Institucionais.

Rikkert et al. ${ }^{20}$, analisando artigos publicados em revistas de geriatria de língua inglesa, notaram que em $29 \%$ havia consentimento informado, em $21 \%$ eram citadas a aprovação prévia por Comitê Institucional e no restante não havia referência aos aspectos éticos. Amdur e Biddle ${ }^{17}$ analisaram as I nstruções aos Autores de 102 revistas ci entíficas de bi omedicina de língua inglesa e observaram que $24 \%$ delas não traziam referências aos aspectos éticos da pesquisa em seres humanos; Rennie e $\mathrm{Yank}^{13}$ realizaram análise informal de 53 artigos sobre consentimento informado e aprovação por Comitê Institucional e obtiveram resultados semel hantes.

A pesar desses autores enfatizarem que há necessidade de incrementar o debate sobre esse aspecto, visando a ampliar e a detal har as orientações rela- tivas à ética na política editorial das revistas científicas médicas e biomédicas, a comparação com os nossos resultados, em que somente $20,8 \%$ das revistas fazem al guma referência à ética da pesquisa em seres humanos, demonstra que as publicações brasileiras estão pouco envolvidas nesse debate.

As Instruções aos Autores das revistas científicas brasileiras, analisadas no presente estudo, enfatizam aspectos técni cos dos artigos, principalmente quanto à forma e às referências bibli ográficas. Esse fato éobservadotambém nos RUMSRB ${ }^{14-16}$, que apesar de incluir aspectos especificamente relacionados à ética, dedicam mai or espaço e detalhamento à técnica dos manuscritos ${ }^{13}$.

Observamos que, nas seis revistas que fazem referências à Declaração de Helsinque, nenhuma contém qual quer orientação sobre como achar esse documento publicado em língua portuguesa ou mesmo em inglês. Realizamos procura informal de cópia atualizada desse documento em português e tivemos dificuldades em obtê-la ${ }^{9,21}$, tornando-se clara a pequena divulgação desse texto no meio científico e editorial brasileiro.

O consentimento do paciente para participar de experimento científico é citado oito vezes nas 139 revistas brasileiras estudadas, sendo que somente em três a redação do texto nas Instruções aos Autores faz referência ao fato de que o consentimento deve ser obtido após o paciente ter sido informado sobre o estudo a ser realizado.

H ossne e Vieira ${ }^{22}$ criticam o termo normal mente usado em língua portuguesa, "consentimento pósinformado", e propõem a redação "consentimento esclareci do", mais adequado ao princí pio de que os indivíduos devem ser não só informados previamente, mas, sim, esclarecidos adequadamente sobre o experimento a ser realizado. A atual norma que regulamenta a pesquisa envolvendo seres humanos no Brasil, Resolução 196/96 do CNS-MS, adota o termo "consenti mento livre e escl areci do".

Os RUMSRB são citados nas Instruções aos Autores de 11 revistas, sendo que em seis as referências são relativas somente aos aspectos técnicos, principalmente relacionados à bibliografia. Somente duas revistas publicaram os RUMRSB em português ${ }^{15,16}$; nove revistas que citam esse documento, fazem referência às publicações de língua inglesa. Somente o J ornal Brasileiro de U rol ogia é claro ao afirmar que os trabal hos devem ser encaminhados de acordo com essas normas, enquanto que as outras revistas afirmam que suas normas são baseadas nos RUMSRB. O J ornal de Pediatria é o único que cita os RUMSRB e, concomitantemente, faz referência explícita às orientações éticas, exigindo aprovação por Comissão de 
Ética e respeito à Resolução 196/96 do CNS-MS.

Esses dados indicam que os RUMSRB é um documento pouco divulgado e conhecido no meio científico brasileiro. Por outro lado, as orientações éticas dos RUMSRB são consideradas extremamente frágei $\mathrm{s}^{13}$ ao soli citarem aos autores que "ao relatar experimentos com seres humanos, indiquem se os procedimentos foram realizados de acordo com os padrões éticos do comitê responsável por experimentação humana (institucional ou regional) e com a Declaração de Helsinque de 1975, tal como revista em 1983"15.

As três revistas que fazem referências genéricas à ética nas suas I nstruções aos Autores utilizam os termos princípios, normas e padrões éticos, não fazendo nenhuma especificação sobre essas orientações e, portanto, constituem orientações éticas frágeis e inconseqüentes.

As 29 revistas que possuem orientações em relação aos aspectos éticos apresentaram comportamento semelhante aos observados por Amdur e Biddle ${ }^{17}$,em relação ao modo como as I nstruções aos Autores solicitam aos pesquisadores que informem sobre os aspectos éticos dos estudos enviados para publicação. A maioria $(57,8 \%)$ pede que as informações sejam incluídas no texto do artigo, geralmente na parte de material e métodos. Por outro lado, o número de revistas que não fazem quaisquer referências a esse aspecto pode ser consi derado el evado $(34,4 \%)$, sugerindo que essas publicações podem não exercer controle adequado sobre os seus próprios critérios para publicação de artigos científicos.

A importância da publicação dos resultados das pesquisas realizadas é, atualmente, inquestionável, tanto do ponto de vista de divulgação do conheci mento científico como em relação aos princípios da éti ca ${ }^{23}$. A publi cação de artigos científicos é um dos principais critérios de avaliação da produtividade dos pesquisadores e das instituições de pesquisa. Portanto, a política editorial das revistas científicas reveste-se de enorme importância no desenvolvimento científico.

A integridade do sistema científico de investigação depende da seriedade dos pesquisadores ${ }^{17}$. No entanto, a política editorial das revistas científicas, objetivando mel horar não só os aspectos técni cos dos artigos, mas também a ética dos estudos, poderia contribuir de modo efetivo para a melhoria global das publicações brasileiras de medicina, biomedicina, odontologia, enfermagem e ciências gerais.

\section{AGRADE CIMENTO}

Os autores agradecem Aparecida Regina Denadai da Silva, bibliotecária, pela obtenção do material desse estudo.

\section{SUMMARY}

\section{Recommendations on ethical aspects inclu- ded in the instructions to authors: analysis of 139 Brazilian Scientific J ournals}

The concern with ethical standards during research in human subjects has been improving in Brazil during the last decade. Analysis of the ethical recommendations in instructions to authors may contribute to understand Brazilian Scientific J ournals policies.

OBJ ECTIVE: to evaluate the recommendations related to the ethics of investigation in human subjects in instructions to authors of Brazilian Scientific J ournals.

MATERIAL AND METHODS: instructions to authors of 139 Brazilian Scientific J ournals in medicine, biomedicine, nursery, odontology and general sciences were analysed.

RESULTS: In one hundred ten (79.1\%) of the 139 journals no recommendations related to ethics werefound; 17 (12.2\%) required previous approval by the institutional committee; $3(2,1 \%)$ the procedures should be in accordance with the Declaration of Helsinki; 1 (0.7\%) recommends the aplication of the informed consent; 5 (3.5\%) recommend the use of U niform requeriments for manuscripts submitted to biomedical journals and 3 (2.1\%) follow no specified rules. Twenty nine journals ask for references in ethics standards: in $15(51.7 \%)$ the information must beincluded in the text; in $2(6,8 \%)$ the authors must send a letter informing how the ethics standards were foll owed; in $1(3.4 \%)$ a copy of the approval by the institutional committee must be included; in $1(3,4 \%)$ bel i eved the authors foll owed the standards and in $10(34.4 \%)$ no specific reference was made.

Concl usion: these results indicated that the majority of Brazilian Scientific J ournals have little concern related to ethical aspects of research in human subjects included in instructions to authors. Even journals that make references to the ethical aspects the recommendations are quite different. [Rev Ass Med Bras 1999; 45(4): 295-302]

KEY WORDS: Ethics. Bioethics. Research. Editoring. J ournals.

\section{REFERÊNCIAS BIBLIOGRÁFICAS}

1. The Nuremberg Code (1947). BMJ 1996; 313: 1.448.

2. World Medical Association - Declaration of Helsinki. J AMA 1997; 277: 925-6.

3. Consel ho Federal de Medicina. Código de Ética Médica 1988.

4. Consel hoF ederal deE nfermagem. Código deÉtica dos Profissionais de Enfermagem 1993. 
5. Conselho Federal de Odontologia. Código de Ética Odontológica 1992.

6. Consel ho Nacional de Saúde - Ministério da Saúde. Normas de Pesquisa em Saúde - Resolução 011988.

7. Consel ho Nacional deSaúde-Ministério da Saúde. Diretrizes e Normas Regulamentadoras de Pesquisa Envolvendo Seres Humanos - Resolução 1961996.

8. Décourt, LV. Ética da investigação clínica. In Gonçalves, EL ed. Pesquisa médica. São Paulo, E.P.U. - CNPq, 1983; 11-23.

9. Vieira, S, Hossne, WS. A ética em pesquisa. In Experimentação com seres humanos, $1^{\text {a }}$ ed. São Paulo; E ditora M oderna, 1987; 51-70.

10. Smith, R. Informed consent: the intricacies. BMJ 1997; 314: 1.059-60.

11. Doyal, L. Informed consent in medical research - J ournals should not publish research to which patients have not given fully informed consent - with three exceptions. BMJ 1997; 314: 1.107-11.

12. Tobias, J S. BMJ 's present policy (sometimes approving research in which patients have not given fully informed consent) is wholly correct. BMJ 1997; 314: 111-4.

13. Rennie, D, Yank, V. Disclosure to the reader of institutional review board approval and informed consent. J AMA 1997; 277: 922-3.

14. International committe of medical journal editors. Uniform requeriments for manuscripts submitted to biomedical journals. J AMA 1997; 277: 927-34.
15. Comitê internacional de editores de revistas médicas. Requisitos uniformes para originais submetidos a revistas biomédicas. J Pediatr 1997; 73: 213-24.

16. Comitê internacional de editores de revistas médicas. Critérios uniformes para encaminhamento de artigos para publicação em jornais deárea biomédica.J Bras U rol 1992; 18: 65-70.

17. Amdur, RJ , Biddle, C. Institutional review board approval and publication of human research results.J AMA 1997; 277: 909-14.

18. Goldenberg, S, Población, DA, Gomes, PO et al. E ditoração de revistas científicas. Análise das instruções aos autores de 19 revistas brasileiras. Acta Cir Bra 1995; 10: 55-60.

19. Brackbill, Y, Hellengers, AE. Ethics and editors. Hasting Cent Rep 1980; 10: 20-4.

20. Rikkert, MGMO, Have, HAMJ , Hoefnagels, WHL. I nformed consent in biomedical studies on aging: survey of four journals. BMJ 1996; 313:1117.

21. Consel ho Federal de Medicina. Resolução 1098 de 30/6/1983 Declaração de Helsinque - Adotada pela $18^{a}$ Assembléia Mundial de Médicos, Helsinque, Finlândia, 1964 e revista na 29a Assembléia Mundial de Médicos, Tóquio, J apão, 1975.

22. Hossne, WS, Vieira, S. Experimentação com seres humanos: aspectos éticos. In Segre, M, Cohen, C. eds. Bioética. São Paulo; EDUSP, 1995; 127-46.

23. Pearn, J . Publication: an ethical imperative. BMJ 1995; 310: 1.313-5. 